\title{
Point contact Andreev reflection by nanoindentation of polymethyl methacrylate
}

\author{
E. Clifford ${ }^{\text {a) }}$ and J. M. D. Coey \\ School of Physics, Trinity College, Dublin 2, Ireland and CRANN, Trinity College, Dublin 2, Ireland
}

(Received 23 February 2006; accepted 13 July 2006; published online 1 September 2006)

\begin{abstract}
A versatile technique for performing spin polarization measurements via point contact Andreev reflection has been developed. This technique involves depositing a superconductor (lead) onto a thin film of the material to be studied through a nanohole formed in a layer of photoresist, using an atomic force microscope as a nanoindenter. Copper and nickel were used to demonstrate the method. The polarizations of $\mathrm{CrO}_{2}$ and $\mathrm{Co}_{2} \mathrm{MnSi}$ were also measured, the former giving a value of $95 \%$, as expected, and the latter giving 20\%, which was surprisingly low for a candidate half metal. () 2006 American Institute of Physics. [DOI: 10.1063/1.2345361]
\end{abstract}

The spin polarization $P$ of a ferromagnetic material is a key property for spin electronics, although the value obtained depends on the measurement technique and the interface involved. ${ }^{1}$ One method for measuring spin polarization is point contact Andreev reflection (PCAR), a process which occurs when a current passes between a normal and a superconducting metal through a ballistic point contact. ${ }^{2}$ For a normal metal, a doubling of the conductance occurs for an applied bias below the superconducting gap energy. However, for spin-polarized materials the Andreev reflection process, and thus the conductance doubling, is suppressed, enabling the value of the spin polarization to be determined. ${ }^{3}$

The standard method for PCAR involves pressing a sharpened niobium tip into a sample and measuring conductance as a function of voltage, below the superconducting transition temperature. While a micrometer system gives enough control to produce ballistic contacts in such situations, it is difficult to define the size of the contact. The size may be estimated from the Sharvin resistance, since the data obtained can only be fitted by using a ballistic model. ${ }^{4}$

Another method involves making a nanohole through a SiN membrane, and then depositing a superconductor and the subject metal on either side of the membrane. ${ }^{5}$ In that case, the use of the membrane as a substrate limits the range of materials that can be studied. Here we have developed a simple and versatile variant method for measuring PCAR in any film or bulk material by means of nanoindentation through a polymethyl methacrylate (PMMA) layer using an atomic force microscope (AFM) tip. Such a technique was originally developed by Bouzehouane et al. to create nanocontacts $^{6}$ and magnetic tunnel nanojunctions. ${ }^{7}$ The method is straightforward to implement and produces robust devices. We show here that it can be used to measure spin polarization in a variety of materials.

A layer of PMMA (Micro-Chem PMMA A2) is spun onto a smooth film of the subject material at $7000 \mathrm{rpm}$ for $1 \mathrm{~min}$, giving an even layer $80 \mathrm{~nm}$ thick [Fig. 1(a)]. The samples were then "soft baked" at $180{ }^{\circ} \mathrm{C}$ for $2 \mathrm{~min}$, so that the PMMA layer is rigid enough to maintain the dimensions of any hole produced, but soft enough to allow the AFM tip to penetrate easily. To create the nanoholes, a Veeco TESP tapping-AFM tip is used in a Digital Instruments multimode

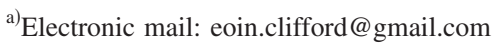

AFM. Such tips have an end diameter of $<15 \mathrm{~nm}$ and are ideal for this application. A sacrificial sample is then used to test the conditions required to create the ideal nanohole, as they will change depending on such factors as the spring constant of the tip, the consistency of the PMMA, etc. The AFM is set to contact mode and a deflection set point is used to press the tip into the sample such that it just passes through the PMMA layer without creating too large a contact [Fig. 1(b)]. The hole can then be imaged by switching the system to tapping mode and scanning the surface; a depth profile of the nanohole is thereby obtained. Though the image formed is a convolution of the tip profile and the hole, which is the same size of the tip, it nevertheless enables us to make an estimate of the hole size.

A layer of lead is then deposited onto the samples using an Edwards Auto 306 evaporation system [Fig. 1(c)]. Interdiffusion between the sample and superconducting layers at the nanocontact is undesirable, as it would modify the composition of the nonsuperconducting sample and lead to increased scattering at the interface. The low melting point of lead $\left(327^{\circ} \mathrm{C}\right)$ reduces the risk of interdiffusion at the nanocontact, as does its large atomic radius. A layer thickness of $100 \mathrm{~nm}$ is used to ensure a good transition temperature $(7.2 \mathrm{~K})$. Kapton tape is placed around the edges before deposition to prevent shorting between the lead layer and the sample.

Electrical contacts are then made on the sample and on the lead layer using silver paint. Conductance curves are obtained using two PerkinElmer 7265 lock-in amplifiers and an Agilent 33120A ac voltage source. One of the lock-in ampli-

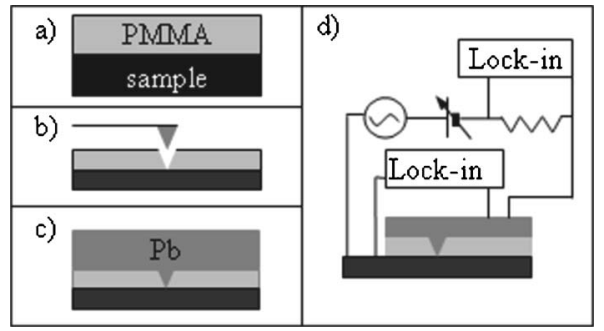

FIG. 1. Fabrication process for PCAR device. (a) A layer of PMMA is spun onto the sample. (b) An AFM tip is used to create a nanohole in the PMMA layer. (c) A superconductor is deposited through the nanohole, creating a nanocontact with the sample. (d) An ac setup is used to measure the conductance across the nanocontact as a function of applied voltage at $4 \mathrm{~K}$. 


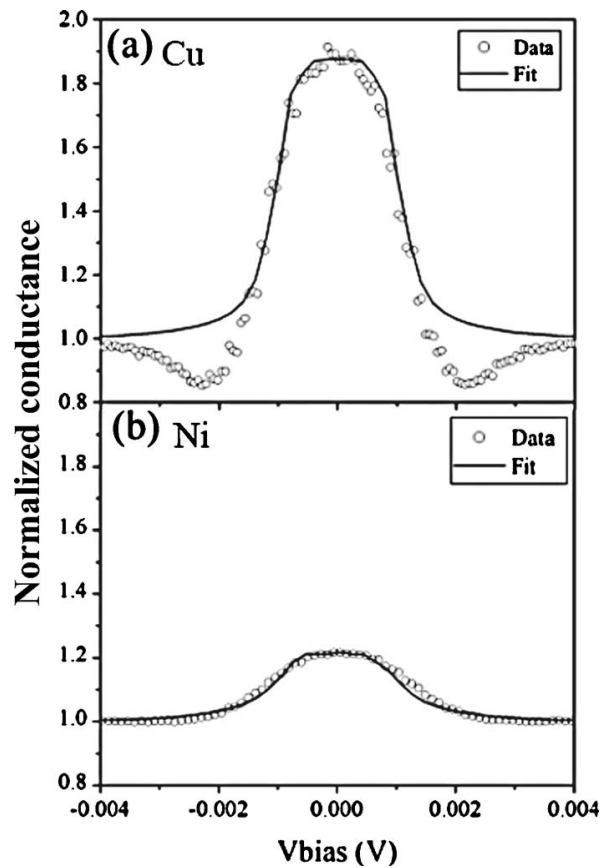

FIG. 2. PCAR measurements. (a) Copper film; the fit used a $Z$ parameter of $0.14, \Delta=1.04 \pm 0.02 \mathrm{meV}$, and zero spin polarization. (b) Nickel film; the fit used a $Z$ parameter of $0.01, \Delta=1.07 \pm 0.02 \mathrm{meV}$, and $P=38 \% \pm 3 \%$.

fiers is used to measure the differential voltage across a shunt resistor, thus effectively measuring the current passing through the nanocontact. The other measures the differential voltage across the nanocontact [Fig. 1(d)]. The area of the contact $A$ can be estimated using the Sharvin formula: ${ }^{8} A$ $=\left(h / 2 e^{2}\right)\left(4 \pi / k_{F}^{2} R\right)$ where $R$ is the resistance of the contact and $k_{F}$ is the Fermi wave vector of the material.

A Keithley 2400 SourceMeter is used to step the bias voltage, and the measurement process is controlled using a LABVIEW program. The sample is cooled to $2 \mathrm{~K}$ in a liquid helium cryostat, well below the transition temperature of lead. Nanocontacts made using this method are extremely stable and an indefinite number of conductance curves can be taken from one sample. These curves are fitted using a modified version of the Blonder-Tinkham-Klapwijk ${ }^{4}$ model developed by Strijkers et al., ${ }^{9}$ with interface scattering accounted for using the $Z$ parameter. A similar model has been developed by Mazin et al. ${ }^{10}$ These two models do not correspond exactly, and the values of spin polarization obtained from both may differ by roughly $5 \%$.

Several materials were studied to establish the validity of the method. Devices were made using $60 \mathrm{~nm}$ films of copper, which were grown on a silicon substrate using a Shamrock sputtering tool. These had a resistance of between 2 and $8 \Omega$, large enough to make any series resistance effect from the copper layer negligible. This corresponds to a contact radius of 6-12 $\mathrm{nm}$, based on the Sharvin formula.

An increase of the conductance is clearly seen below the superconducting gap [Fig. 2(a)]. A fit of the data gave a value of 0.14 for $Z$, with no spin polarization. Dips were observed around the superconducting gap. These are caused by the proximity effect, a process where Cooper pairs leak from a superconductor into an adjacent metal, creating a thin superconducting layer in the normal metal. ${ }^{9}$ A value of $1.04 \pm 0.02 \mathrm{meV}$ for the superconducting gap was used in the fit, less than the $1.25 \mathrm{meV}$ expected for lead at $2 \mathrm{~K}$. It is Downloaded 12 Aug 2009 to 134.226.1.229. Redistribution subject

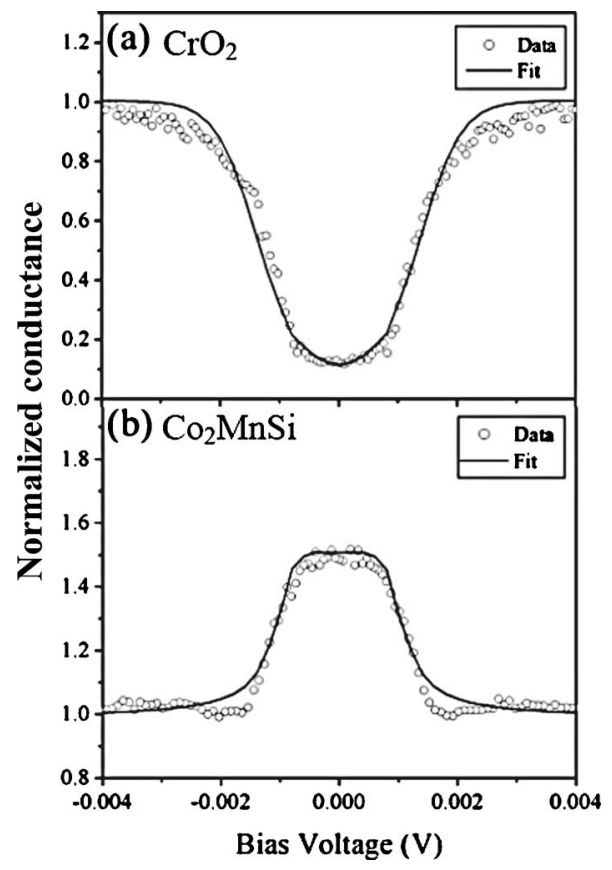

FIG. 3. PCAR measurements. (a) $\mathrm{CrO}_{2}$ film; the fit used a $Z$ parameter of $0.11, \Delta=1.30 \pm 0.02 \mathrm{meV}$, and $P=95 \% \pm 2 \%$. (b) $\mathrm{Co}_{2} \mathrm{MnSi}$ film; the fit used a $Z$ parameter of $0.12, \Delta=1.05 \pm 0.02 \mathrm{meV}$, and a spin polarization of $20 \% \pm 3 \%$

possible that this reduction is an artifact of the fitting procedure used.

Devices were also made using a ferromagnetic material, nickel, as the base layer. $60 \mathrm{~nm}$ films were grown on a silicon substrate using a Shamrock sputtering machine. A conductance curve is shown in Fig. 2(b) for a sample with a resistance of $14 \Omega$. The data show a suppression of the subgap doubling caused by the spin polarization of the material. A value of $P=38 \% \pm 3 \%$ is inferred from a fit to the data, close to reported polarization values obtained from previous PCAR experiments $(32 \%-37 \%))^{5,9}$ Again the fitted value for the superconducting gap, $1.07 \pm 0.02 \mathrm{meV}$, was less than that expected for lead. Substituting the resistance into the Sharvin formula gives a radius of $3.8 \mathrm{~nm}$ for the contact.

Two proposed half metals were then examined using this method. Films of the type IA (Ref. 11) half metal $\mathrm{CrO}_{2}$ were made by thermal decomposition of $\mathrm{CrO}_{3}$ under natural oxygen pressure in a sealed vessel in the presence of (100) oriented $\mathrm{TiO}_{2}$ substrates. ${ }^{12}$ The data show a drop in conductance, indicating a high degree of spin polarization [Fig. $3(\mathrm{a})$ ]; fitting gave a value of $95 \% \pm 2 \%$ which compares well with reported values of $96 \% .^{13}$ A value of $1.30 \pm 0.02 \mathrm{meV}$ was obtained for the superconducting gap. This particular sample had a resistance of $37 \Omega$. Taking a value of 2.17 $\times 10^{9} \mathrm{~m}^{-1}$ for the Fermi wave vector of $\mathrm{CrO}_{2}$ gives us a radius of $17 \mathrm{~nm}$ from the Sharvin formula.

The second half metal was the Heusler alloy $\mathrm{Co}_{2} \mathrm{MnSi}$. This compound is predicted to be a type IA half metal, ${ }^{11}$ with density of states calculations showing an energy gap of $0.4 \mathrm{eV}$ in the minority band. ${ }^{14}$ Films of $\mathrm{Co}_{2} \mathrm{MnSi}$ were grown by sputtering onto a silicon substrate and postannealed under vacuum at $500{ }^{\circ} \mathrm{C}$ for $2 \mathrm{~h}$. X-ray diffraction analysis confirmed the $L 2{ }_{1}$ Heusler structure. Conductance curves showed some suppression of the Andreev process [Fig. 3(b)], but a fit of the data only gives a spin polarization of $20 \% \pm 3 \%$. Once again quite a low $Z$ value $(0.12)$ was to AIP license or copyright; see http://apl.aip.org/apl/copyright.jsp 
used in the fit. A value of $1.05 \pm 0.02 \mathrm{meV}$ was obtained for the superconducting gap.

The measured polarization value for $\mathrm{Co}_{2} \mathrm{MnSi}$ was surprising for a material predicted to be fully half metallic. This result could be attributed to antisite disorder between the Co and $\mathrm{Mn}$ atoms, which have a similar atomic radius. Studies have shown Co-Mn antisite occupancy of up to $7 \%$ for this material. ${ }^{15}$ While values of up to $61 \%$ have been reported elsewhere, ${ }^{16} 100 \%$ polarization has never been observed for this material; a recent study by Wang et al. estimated a polarization value of only $12 \%$ at the Fermi level using spinresolved photoemission spectra. ${ }^{17}$

The nanoindentation PCAR method is a straightforward technique which enables spin polarization to be measured for materials in the as-prepared state, on surfaces exposed to ambient conditions. It gives results for $\mathrm{Cu}, \mathrm{Ni}$, and $\mathrm{CrO}_{2}$ which are similar to those obtained by the standard method. It has many advantages compared to the standard niobium tip method. The samples are robust and can be measured several times, and then stored and remeasured. The profile of the hole matches that of the AFM tip, and imaging supports this. Most of the samples produced by this method gave a low $Z$ value, indicating a good quality interface. Higher $Z$ values lead to more spin-scattering events at the interface and reduce the measured polarization. ${ }^{18}$

This work was supported by Science Foundation Ireland, as part of the CINSE program.
${ }^{1}$ I. I. Mazin, Phys. Rev. Lett. 83, 1427 (1999).

${ }^{2}$ A. F. Andreev, Zh. Eksp. Teor. Fiz. 46, 1823 (1964) [Sov. Phys. JETP 19, 1228 (1964)].

${ }^{3}$ R. J. Soulen, J. M. Byers, M. S. Osofsky, B. Nadgorny, T. Ambrose, S. F. Cheng, P. R. Broussard, C. T. Tanaka, J. Nowak, J. S. Moodera, A. Barry, and J. M. D. Coey, Science 282, 85 (1998).

${ }^{4}$ G. E. Blonder, M. Tinkham, and T. M. Klapwijk, Phys. Rev. B 25, 4515 (1982).

${ }^{5}$ S. K. Upadhyay, A. Palanisami, R. N. Louie, and R. A. Buhrman, Phys. Rev. Lett. 81, 3247 (1998).

${ }^{6}$ K. Bouzehouane, S. Fusil, M. Bibes, J. Carrey, T. Blon, M. Le Dû, P. Seneor, V. Cros, and L. Vila, Nano Lett. 3, 1599 (2003).

${ }^{7}$ J. Carrey, K. Bouzehouane, J. M. George, C. Ceneray, T. Blon, M. Bibes, A. Vaurès, S. Fusil, S. Kenane, L. Vila, and L. Piraux, Appl. Phys. Lett. 81, 760 (2002).

${ }^{8}$ Y. V. Sharvin, Sov. Phys. JETP 21, 655 (1965).

${ }^{9}$ G. J. Strijkers, Y. Ji, F. Y. Yang, C. L. Chien, and J. M. Byers, Phys. Rev. B 63, 104510 (2001).

${ }^{10}$ I. I. Mazin, A. A. Golubov, and B. Nadgorny, J. Appl. Phys. 89, 7576 (2001).

${ }^{11}$ J. M. D. Coey and M. Venkatesan, J. Appl. Phys. 91, 8345 (2002).

${ }^{12}$ L. Ranno, A. Barry, and J. M. D. Coey, J. Appl. Phys. 81, 5774 (1997).

${ }^{13}$ Y. Ji, G. J. Strijkers, F. Y. Yang, C. L. Chien, J. M. Byers, A. Anguelouch, Gang Xiao, and A. Gupta, Phys. Rev. Lett. 86, 5585 (2001).

${ }^{14}$ S. Ishida, T. Masaki, S. Fujii, and S. Asano, Physica B 245, 1 (1998).

${ }^{15}$ B. Ravel, M. P. Raphael, V. G. Harris, and Q. Huang, Phys. Rev. B 65, 184431 (2002)

${ }^{16}$ S. Kämmerer, A. Thomas, A. Hütten, and G. Reiss, Appl. Phys. Lett. 85, 79 (2004).

${ }^{17}$ W. H. Wang, M. Przybylski, W. Kuch, L. I. Chelaru, J. Wang, Y. F. Lu, J. Barthel, H. L. Meyerheim, and J. Kirschner, Phys. Rev. B 71, 104416 (2005).

${ }^{18}$ C. H. Kant, O. Kurnosikov, A. T. Filip, H. J. M. Swagten, and W. J. M. de Jonge, J. Appl. Phys. 93, 7528 (2003). 